\title{
MAPK4 silencing together with a PARP1 inhibitor as a combination therapy in triple-negative breast cancer cells
}

\author{
XIAOQI ZENG ${ }^{1 *}$, SHANSHAN JIANG $^{2 *}$, SIMIN RUAN $^{1 *}$, ZHAOZE GUO $^{1}$, \\ JINGYUN GUO $^{1}$, MINFENG LIU ${ }^{1}$, CHANGSHENG YE ${ }^{1}$ and JIANYU DONG ${ }^{1}$ \\ ${ }^{1}$ Breast Center, Department of General Surgery, Nanfang Hospital, Southern Medical University, \\ Guangzhou, Guangdong 510515; ${ }^{2}$ Institute of Hematological Research, \\ Shaanxi Provincial People's Hospital, Xi'an, Shaanxi 710000, P.R. China
}

Received December 14, 2020; Accepted April 23, 2021

DOI: $10.3892 / \mathrm{mmr} .2021 .12187$

\begin{abstract}
Triple-negative breast cancer (TNBC) is the most common type of cancer among females worldwide and is associated with poor prognosis. Poly ADP-ribose polymerase-1 (PARP1) inhibitors are effective against TNBC with mutations in the breast cancer type 1 susceptibility protein (BRCA1) and/or BRCA2 genes; however, the development of resistance to PARP1 inhibitors limits their use. Thus, identifying strategies to overcome this resistance is urgently required. The aim of the present study was to investigate the potential function and mechanism of small interfering (si)RNA-MAPK4 (siMAPK4) in enhancing the efficacy of a PARP1 inhibitor and reducing the resistance. In the present study, data on the mRNA expression level of MAPK4 in normal breast tissues and TNBC tissues were obtained from The Cancer Genome Atlas database. The mRNA and protein expression levels of MAPK4 in normal breast cells and TNBC cells were analyzed using reverse transcription-quantitative PCR and western blotting, respectively. The phosphorylated (p) histone $\mathrm{H} 2 \mathrm{AX}(\gamma \mathrm{H} 2 \mathrm{AX})$ protein expression was assessed via immunofluorescence. Cell Counting Kit-8, wound healing and TUNEL assays were used to determine the proliferative, migratory and apoptotic abilities
\end{abstract}

Correspondence to: Dr Jianyu Dong or Dr Changsheng Ye, Breast Center, Department of General Surgery, Nanfang Hospital, Southern Medical University, 1838 North Guangzhou Avenue, Guangzhou, Guangdong 510515, P.R. China

E-mail: dongjy@smu.edu.cn

E-mail: smuyecs@163.com

${ }^{*}$ Contributed equally

Abbreviations: TNBC, triple-negative breast cancer; PARP1, poly ADP-ribose polymerase-1; BRCA1/2, breast cancer type 1 and 2 susceptibility protein; TCGA, The Cancer Genome Atlas; AKT-CA, constitutively active AKT; RT-qPCR, reverse transcription-quantitative PCR; casp-3, caspase-3

Key words: TNBC, MAPK4, PARP1 inhibitor, AKT phosphorylation, DNA repair of $\mathrm{HCC} 1937$ cells. MAPK4 was highly expressed in TNBC patient tissues and cell lines. Moreover, overexpression of MAPK4 could promote HCC1937 cell proliferation. Treatment of HCC1937 cells with the combination of siMAPK4 and a PARP1 inhibitor olaparib decreased their proliferation and migration and increased their apoptosis. The protein expression levels of the DNA repair-related proteins p-DNA-dependent protein kinase catalytic subunit (DNA-PK) and RAD51 recombinase (RAD51) were inhibited in the siMAPK4 and siMAPK4 + olaparib groups. However, the marker of a double-stranded break $\gamma \mathrm{H} 2 \mathrm{AX}$ showed increased protein expression in the siMAPK4 + olaparib group. As MAPK4 could phosphorylate AKT at threonine $308\left(\mathrm{AKT}^{\mathrm{T} 308}\right.$ ), the current study restored $\mathrm{p}-\mathrm{AKT}^{\mathrm{T} 308}$ using a constitutively active AKT plasmid (AKT-CA). p-DNA-PK and RAD51 showed high expression and $\gamma \mathrm{H} 2 \mathrm{AX}$ exhibited lower protein expression in the AKT-CA group. The present findings suggested that siMAPK4 can enhance the sensitivity of TNBC cells to PARP1 inhibitors.

\section{Introduction}

Breast cancer is the most commonly diagnosed type of cancer and the third leading cause of cancer-related mortality among females worldwide (1). In 2020, 2.65 million new cases and 685,000 deaths occurred, accounting for the second highest incidence of cancer and fifth highest rate of cancer-related mortality (2). Moreover, the incidence and mortality rates of breast cancer are rising annually (2). Triple-negative breast cancer (TNBC), which is defined by a lack of expression of estrogen receptor, progesterone receptor and HER-2, accounts for $11-20 \%$ of all breast carcinomas $(3,4)$. Notably, targeted therapy has a poor effect on TNBC (5). Therefore, the discovery of novel therapies for the treatment of TNBC is urgently required.

MAPK4, also termed ERK4, p63 and Prkm4, is an atypical MAPK as it is not phosphorylated by the dual serine/threonine and tyrosine MAPK kinase due to a lack of the threonine-X-tyrosine activation motif (6). Thus far, MAPK4 has been associated with several diseases, such as numerous types of cancer and methamphetamine addiction leading to psychosis (7). According to The Cancer Genome 
Atlas (TCGA), lung adenocarcinoma, low-grade glioma, thyroid carcinoma and urothelial carcinoma with upregulation of MAPK4 are associated with poor prognosis (8). Moreover, MAPK4 phosphorylates AKT at threonine $308\left(\mathrm{AKT}^{\mathrm{T} 308}\right)$ and serine $473\left(\mathrm{AKT}^{\mathrm{S} 473}\right)$, thereby promoting cell proliferation and inhibiting apoptosis (9). It has been verified that microRNA (miR)-767-5p upregulates MAPK4, and that the circular (circ)RNA circ_0000190 inhibits the progression of multiple myeloma by regulating MAPK4 (10). Furthermore, circRNA MAPK4 inhibits apoptosis by regulating miR-125a-3p, which inhibits the MAPK signaling pathway (11). This evidence indicates the role of MAPK4 as an oncogene. It has been shown that the AKT signaling pathway is associated with the DNA damage repair mechanism $(12,13)$. Hence, we hypothesized that MAPK4 could regulate DNA damage repair by activating the AKT signaling pathway.

In recent years, the DNA damage repair mechanism has been utilized in the treatment of cancer (14). Poly ADP-ribose polymerase-1 (PARP1) serves vital roles in DNA damage repair of single-strand and double-strand breaks by catalyzing the formation of poly ADP-ribose and maintaining genome integrity (15). PARP inhibitors, such as olaparib and rucaparib, have been approved by the US Food and Drug Administration, and it has been shown that these drugs are effective for the treatment of ovarian and breast cancer, particularly TNBC (16,17). However, the wide use of PARP inhibitors can lead to the development of resistance (18). Thus, there is an urgent requirement to discover novel targets or drugs that can be combined with PARP1 inhibitors.

As aforementioned, MAPK4 was identified to be associated with several cancer types, and can promote DNA repair by increasing AKT phosphorylation. Thus, small interfering (si)RNA MAPK4 (siMAPK4) may be a novel molecule to combine with PARP1 inhibitors. Therefore, the present study aimed to investigate the potential function and mechanism of siMAPK4 in enhancing the efficacy of a PARP1 inhibitor, which could reduce the possibility of PARP1 inhibitor resistance in TNBC and provide a new therapeutic strategy for the treatment of TNBC.

\section{Materials and methods}

Materials and reagents. RPMI-1640 medium (cat. no. 11875093), FBS (cat. no. 10100139C) and $100 \mathrm{IU} / \mathrm{ml}$ penicillin-streptomycin (cat. no. 15140-122) were purchased from Gibco; Thermo Fisher Scientific, Inc. MCF-10A-specific medium (cat. no. CM-0525) was from Procell Life Science \& Technology Co., Ltd. The PARP1 inhibitor olaparib (cat. no. HY-10162) was purchased from MedChemExpress. Antibodies against MAPK4 (cat. no. ab211501), p-AKT ${ }^{\mathrm{T} 308}$

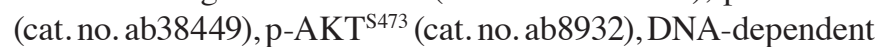
protein kinase catalytic subunit (DNA-PK; cat. no. ab44815), p-DNA-PK (cat. no. ab18192), RAD51 (cat. no. ab63801), Bcl-2 (cat. no. ab59348), Bax (cat. no. ab53154), cleaved-casp-3 (cat. no. ab32042) and GAPDH (cat. no. ab245355) were from Abcam, while HRP-labeled goat anti-mouse and anti-rabbit IgG (cat. nos. ZB-2301 and ZB-2305, respectively) were from OriGene Technologies, Inc. The anti-p-histone H2AX ( $\gamma$ H2AX; phospho-Ser139; cat. no. K001453M) antibody was purchased from Beijing Solarbio Science \& Technology Co.,
Ltd., while the anti-mouse $\operatorname{IgG}(\mathrm{H}+\mathrm{L}) \mathrm{F}(\mathrm{ab}) 2$ Fragment (Alexa Fluor ${ }^{\circledR} 488$ Conjugate; cat. no. 4408S) for immunofluorescence was from Cell Signaling Technology, Inc. The RNeasy Mini kit (cat. no. 74104), QuantiTect Rev Transcription kit (cat. no. 205311) and miScript SYBR Green PCR kit (cat. no. 218073) were obtained from Qiagen GmbH. siMAPK4-1 (5'-GGUGAGCUGUUC AAGUUCAGC-3'), siMAPK4-2 (5'-GGA AGGUCG CUGUGAAGAAGA-3'), siMAPK4-3 (5'-GGUUGGUAA CAAAGUGGUACC-3') and scramble siRNA (5'-GGCGTG AATGGGTCTAACCTT-3') were purchased from Addgene, Inc. Overexpression-MAPK4 plasmid (cat. no. C05008), the pcDNA 3.1 empty vector (cat. no. C06002), which was used as the overexpression-negative control (NC), and constitutively active AKT (AKT-CA) plasmids (cat. no. 53583) were designed and purchased from Shanghai GenePharma Co., Ltd. The mRNA expression level of MAPK4 from 116 TNBC samples and 114 normal samples was obtained from UALCAN (http://ualcan.path.uab.edu) (19), which could provide mRNA expression data regarding cancer and normal samples in TCGA.

Cell culture. MCF-10A (normal human mammary epithelial cell line) cells were purchased from The Cell Bank of Type Culture Collection of Chinese Academy of Sciences and cultured in MCF-10A-specific medium (Procell Life Science \& Technology Co., Ltd.). MDA-MB-468 and MDA-MB-231 (breast adenocarcinoma), HCC1937 (ductal breast carcinoma) cells are TNBC cell lines (all from The Cell Bank of Type Culture Collection of Chinese Academy of Sciences) and were cultured in RPMI-1640 medium containing 10\% FBS and $1 \% 100 \mathrm{IU} / \mathrm{ml}$ penicillin-streptomycin at $37^{\circ} \mathrm{C}$ in an incubator with $5 \% \mathrm{CO}_{2}$.

Cell transfection. According to the instructions provided by the manufacturer of Lipofectamine ${ }^{\circledR} 2000$ (Thermo Fisher Scientific, Inc.), HCC1937 cells were seeded in 6-well plates at the density of $1.2 \times 10^{6}$ cells/well. The following day, $10 \mu 1$ Lipofectamine 2000 and $4 \mu \mathrm{g}$ plasmids were diluted with $500 \mu 1$ Opti-MEM (Thermo Fisher Scientific, Inc.) and incubated at room temperature for $20 \mathrm{~min}$. Subsequently, the Lipofectamine 2000 and plasmids solution was added to the cells. Following $6 \mathrm{~h}$ of incubation at $37^{\circ} \mathrm{C}$, the medium was changed with fresh RPMI-1640 medium. After $24 \mathrm{~h}$, the subsequent experiments were performed with the transfected HCC1937 cells.

Western blotting. Western blotting was performed as previously described (20,21). Briefly, total protein of HCC1937 cells was extracted using RIPA lysis buffer (Beyotime Institute of Biotechnology), quantified using an Enhanced BCA Protein assay kit (Beyotime Institute of Biotechnology), separated via 10\% SDS-PAGE (20 $\mu \mathrm{g}$ total protein/lane) and transferred to nitrocellulose membranes (Whatman pcl). After blocking with $5 \%$ fat-free milk for $1 \mathrm{~h}$ at room temperature, the membranes were incubated with primary antibodies at $4^{\circ} \mathrm{C}$ overnight. After washing three times with TBS-Tween-20 (1\%), the membranes were incubated with secondary antibodies for $1 \mathrm{~h}$ at room temperature. Finally, the blots were acquired using Supersignal WestFemto Maximum Sensitivity substrate 
(Thermo Fisher Scientific, Inc.) and a ChemiDoc XRS ${ }^{+}$system (Bio-Rad Laboratories). The antibodies used were as follows: MAPK4 (1:1,000), p-AKT ${ }^{\mathrm{T} 308}$ (1:800), p-AKT ${ }^{\mathrm{S} 473}(1: 800)$, DNA-PK $(1: 1,000)$, p-DNA-PK $(1: 200)$, RAD51 $(1: 3,000)$ Bcl-2 (1:800), Bax (1:800), cleaved-casp-3 (1:800), GAPDH $(1: 2,000)$, and HRP-labeled goat anti-mouse and anti-rabbit $\operatorname{IgG}(1: 2,000)$.

MTT assay. The MTT assay was performed as previously described (21). Briefly, HCC1937 cells transfected with siMAPK, overexpression-MAPK4 or scramble siRNA were seeded into 96-well plates (5,000 cells/well), and $10 \mu \mathrm{M}$ olaparib was added the following day for $24 \mathrm{~h}$. After incubating for 24,48 and $72 \mathrm{~h}$ in a $37^{\circ} \mathrm{C}$ incubator, cells were incubated with MTT $(10 \mu \mathrm{g} / \mathrm{ml})$ at $37^{\circ} \mathrm{C}$ for $4 \mathrm{~h}$, then $150 \mu \mathrm{l}$ DMSO was added to the wells and the optical density values were measured.

Wound healing assay. The wound healing assay was performed as previously described (20,21). Briefly, HCC1937 cells transfected with siMAPK4 or scramble siRNA, and control HCC1937 cells were seeded into 6-well plates at the density of $10^{6}$ cells per well, which had been marked with a straight line on the back of the plates in advance. The following day, cells were treated with $10 \mu \mathrm{M}$ olaparib for $24 \mathrm{~h}$ in a $37^{\circ} \mathrm{C}$ incubator. Following the treatment, wounds were made in the cell monolayer by scratching with $10-\mu l$ pipette tips. The cells were then incubated in DMEM without FBS at $37^{\circ} \mathrm{C}$ in an incubator. Images were captured using a light microscope (magnification, x 100) at 0 and $24 \mathrm{~h}$ after treatment with olaparib and the migratory distance was measured using ImageJ 1.49 (National Institutes of Health).

Immunofluorescence. Immunofluorescence was performed as previously described (21). Briefly, cells were fixed in $4 \%$ paraformaldehyde for $15 \mathrm{~min}$ at room temperature and blocked with 2\% BSA (Sigma-Aldrich; Merck KGaA) for $1 \mathrm{~h}$ at room temperature. Primary antibody $\gamma \mathrm{H} 2 \mathrm{AX}$ (1:200) was incubated at $4^{\circ} \mathrm{C}$ overnight and FITC-labelled secondary anti-mouse antibody (1:400; cat. no. 4408S; Cell Signaling Technology, Inc.) was incubated for $1 \mathrm{~h}$ at room temperature. Nuclei were counterstained with DAPI at room temperature. Finally, cells were sealed, and images were acquired using a confocal microscope (magnification, x100).

$R T$-quantitative $(R T-q) P C R$. mRNA was extracted using a RNeasy Mini kit and reverse transcribed into cDNA according to the manufacturer's instructions of the QuantiTect Rev Transcription kit. qPCR was performed with the miScript SYBR Green PCR kit. The system was as follows: $10 \mu 12 \mathrm{X}$ SYPR Green Supermix, $1 \mu 1$ forward primer, $1 \mu 1$ reverse primer, $2 \mu \mathrm{l} \mathrm{cDNA}$ and $6 \mu \mathrm{l}$ RNase-free water. The amplification was performed as follows: Initial denaturation at $95^{\circ} \mathrm{C}$ for $10 \mathrm{~min}$; followed by 40 cycles of $95^{\circ} \mathrm{C}$ for $15 \mathrm{sec}, 60^{\circ} \mathrm{C}$ for $30 \mathrm{sec}$; and a final extension at $72^{\circ} \mathrm{C}$ for $30 \mathrm{sec}$. The mRNA expression levels were quantified using the $2^{-\Delta \Delta C q}$ method (22). The primers used were as follows: MAPK4 forward, 5'-ATC CTGGCTGAGATGCTTAC-3' and reverse, 5'-CTTGTC TTCCTCCCGGATTAC-3' (product length, $108 \mathrm{bp}$ ); and GAPDH (NM_001289726.1) forward, 5'-CCTTCCGTGTTC
CTACCC-3' and reverse, 5'-AAGTCGCAGGAGACAACC-3' (product length, 163 bp); and AKT (NM_001382432) forward, 5'-TGGACTACCTGCACTCGGAGAA-3' and reverse, 5'-GTG CCGCAAAAGGTCTTCATGG-3' (product length, 154 bp).

TUNEL assay. The TUNEL assay was performed according to the manufacturer's instructions (Roche Diagnostics). Cells were fixed with $4 \%$ paraformaldehyde for $25 \mathrm{~min}$ at room temperature and washed twice with PBS. Subsequently, $0.2 \%$ Triton $\mathrm{X}-100$ was added for $5 \mathrm{~min}$ at room temperature, the cells were washed with PBS and TUNEL reaction reagent was added for $60 \mathrm{~min}$ at $37^{\circ} \mathrm{C}$ in a dark moisture chamber. Of note, only dUTP solution and DNase 1 were added to the negative and positive control groups, respectively. After the slides were washed, converter-POD was added for $30 \mathrm{~min}$ at $37^{\circ} \mathrm{C}$. The cells were washed again thrice with PBS, and diaminobenzidine was added at room temperature for $10 \mathrm{~min}$. Next, the slides were washed with PBS, captured and counterstained with hematoxylin for $1 \mathrm{~min}$ at room temperature, washed with running water and dehydrated with gradient alcohol (50, 70, 85, 95 and 100\%) for $1 \mathrm{~min}$ at each concentration. The process was completed by the addition of xylene twice ( 5 min per time) and sealing of the slides with neutral gum (Beijing Solarbio Science \& Technology Co., Ltd.). The apoptotic rate was calculated in 200 cells obtained from different groups using a light microscope (magnification, x100) (23).

Statistical analysis. All experiments were repeated in triplicate and data are presented as the mean \pm SEM, and statistical analyses were performed using GraphPad Prism 5.01 software (GraphPad Software, Inc.). An unpaired t-test and one-way ANOVA were used to determine the significant differences, and multiple comparison between the groups was performed using Bonferroni's method. $\mathrm{P}<0.05$ was considered to indicate a statistically significant difference.

\section{Results}

MAPK4 is highly expressed in TNBC tissues and cell lines. MAPK4 is upregulated in numerous types of cancer, including lung adenocarcinoma and bladder cancer (9). By analyzing 114 normal breast tissues and 116 TNBC samples from TCGA database, it was found that TNBC samples had higher MAPK4 mRNA expression compared with normal samples (Fig. 1A). Moreover, the TNBC cell lines MDA-MB-468, HCC1937 and MDA-MB-231 expressed higher mRNA and protein levels of MAPK4 compared with the normal MCF-10A cell line (Fig. 1B and C). These findings indicated that the upregulation of MAPK4 may be associated with TNBC.

MAPK4 promotes the proliferation of HCC1937 cells. HCC1937 cells were transfected with siMAPK4 and an overexpression-MAPK4 plasmid. As HCC1937 cells expressed higher MAPK4 expression, these cells were chosen for knockdown experiments. The siMAPK4 group had lower MAPK4 mRNA and protein expression, while the overexpression-MAPK4 group had higher MAPK4 expression compared with the control groups (HCC1937 wild-type cells) (Fig. 2A-D). Then their proliferation was determined and the results revealed 
A

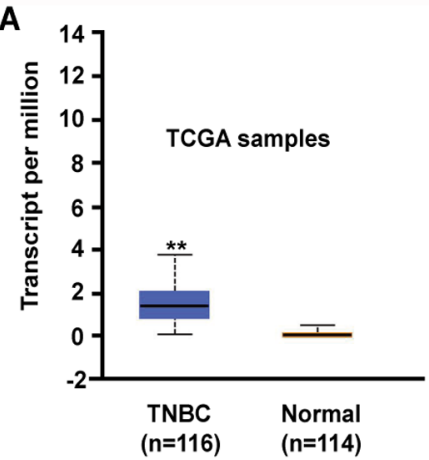

B

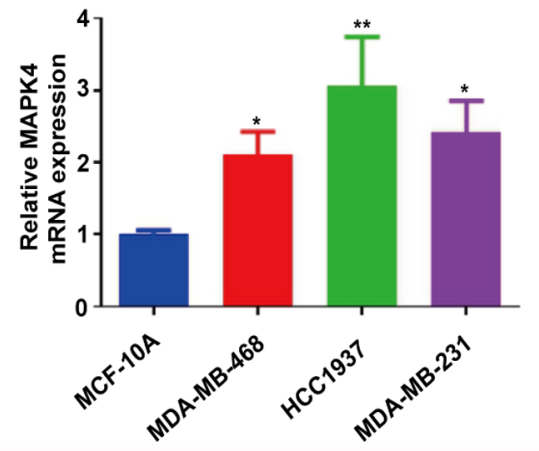

C

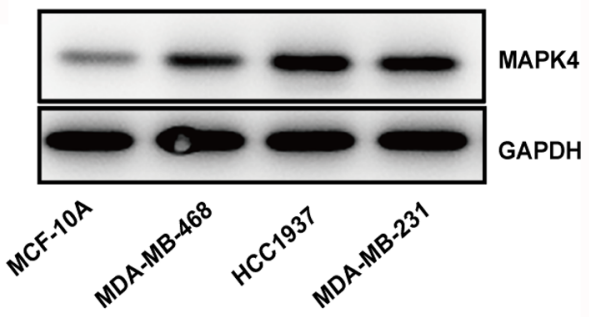

Figure 1. MAPK4 is highly expressed in TNBC tissues and cell lines. (A) MAPK4 mRNA expression in 114 normal breast tissues and 116 TNBC samples of different subclasses of breast cancer from TCGA. ${ }^{* *} \mathrm{P}<0.01 \mathrm{vs}$. Normal group. Relative (B) mRNA and (C) protein expression levels of MAPK4 in MCF-10A, MDA-MB-468, HCC1937 and MDA-MB-231 cells. ${ }^{*} \mathrm{P}<0.05,{ }^{* *} \mathrm{P}<0.01$ vs. MCF-10A cells. TCGA, The Cancer Genome Atlas; TNBC, triple-negative breast cancer.

A

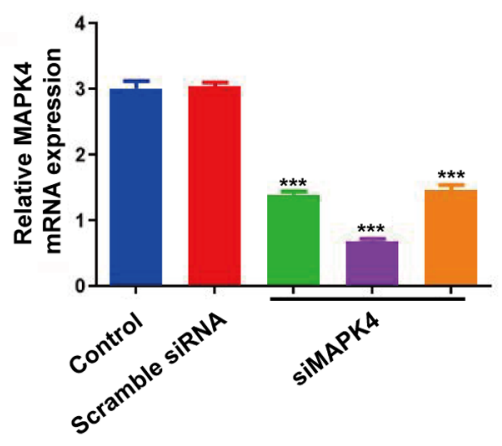

D

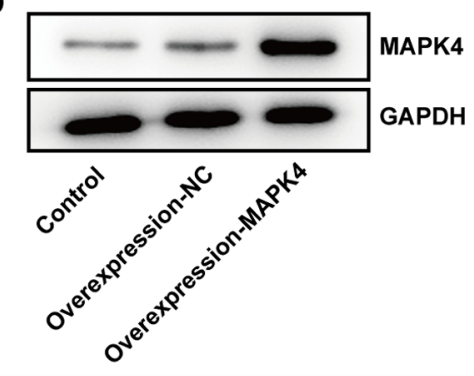

B
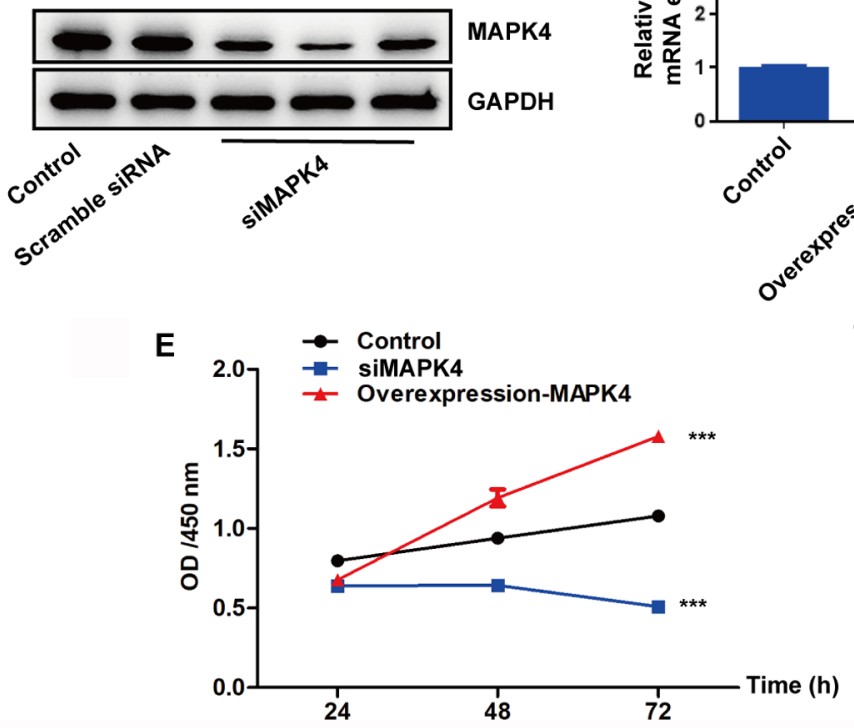

C

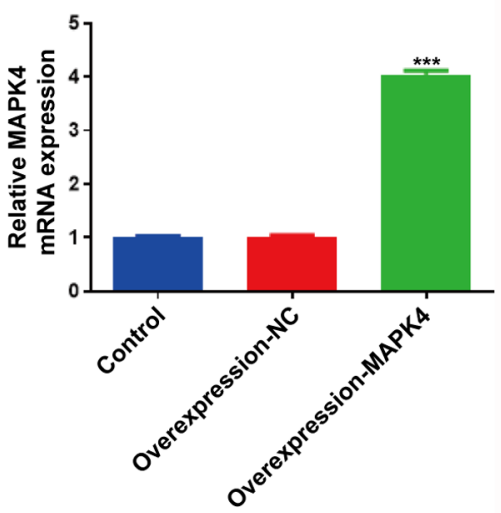

Figure 2. MAPK4 promotes the proliferation of HCC1937 cells. (A) Extraction of total mRNA from siMAPK4 cells, scramble siRNA cells and control cells. RNA was reverse transcribed to cDNA, and the relative mRNA expression levels of MAPK4 were determined via RT-qPCR. (B) The total protein of siMAPK4 cells, scramble siRNA cells and control cells was extracted, and the expression level of MAPK4 was measured via western blotting. (C) Extraction of total mRNA from control cells, overexpression-NC cells and overexpression-MAPK4 cells. RNA was reverse transcribed to cDNA, and the relative mRNA expression levels of MAPK4 were determined using RT-qPCR. (D) The total protein of control cells, overexpression-NC cells and overexpression-MAPK4 cells was extracted, and the expression level of MAPK4 was measured via western blotting. (E) Control cells, overexpression-MAPK4 cells and siMAPK4 cells were seeded in 96-well plates (5,000 cells/well). MTT assays were performed at 24, 48 and $72 \mathrm{~h} .{ }^{* * *} \mathrm{P}<0.001$ vs. control. NC, negative control; OD, optical density; RT-qPCR, reverse transcription-quantitative PCR; siRNA, small interfering RNA; siMAPK4, siRNA-MAPK4.

that knockdown and overexpression of MAPK4 inhibited and promoted cell proliferation, respectively, compared with the control group (Fig. 2E).

siMAPK4 enhances the sensitivity of TNBC cells to olaparib, a PARPl inhibitor. Next, it was investigated how silencing of MAPK4 could affect the efficacy of the PARP1 inhibitor, olaparib. For this purpose, HCC1937 cells were treated with $10 \mu \mathrm{M}$ olaparib and siMAPK4 separately or in combination at $37^{\circ} \mathrm{C}$ in an incubator. Both siMAPK and olaparib inhibited cell proliferation and migration, and promoted apoptosis.
Moreover, as expected, the combination groups showed increased toxicity (Fig. 3A-E).

MAPK4 exerts its DNA damage repair function by regulating the AKT signaling pathway. A previous study reported that MAPK4 could promote tumor progression by phosphorylating $\mathrm{AKT}^{\mathrm{S} 473}$ and $\mathrm{AKT}^{\mathrm{T} 308}$ to exert its DNA damage and repair function (9). To verify this, the phosphorylation of $\mathrm{AKT}^{\mathrm{S} 473}$ and $\mathrm{AKT}^{\mathrm{T} 308}$ was detected in HCC1937 cells. p-AKT ${ }^{\mathrm{T} 308}$ expression was markedly decreased in the siMAPK4 group, while no significant differences were observed in $\mathrm{p}-\mathrm{AKT}^{\mathrm{S} 473}$ expression between the 
A

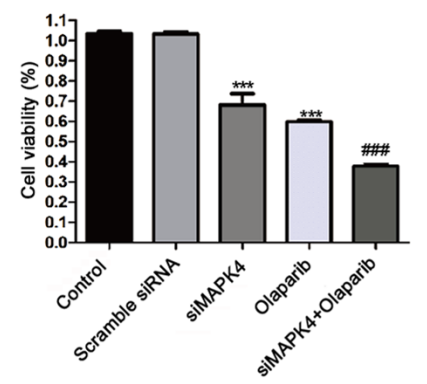

B

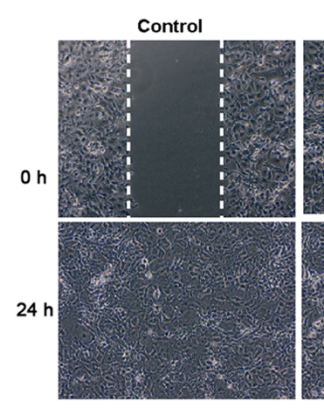

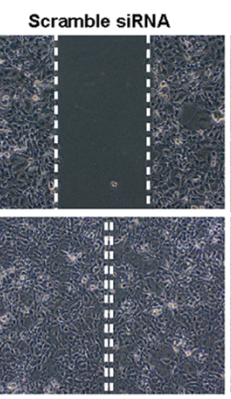

SiMAPK4

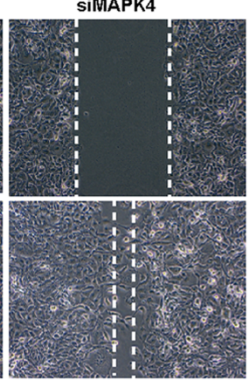

Olaparib

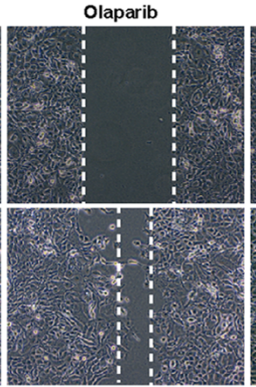

siMAPK4+Olaparib

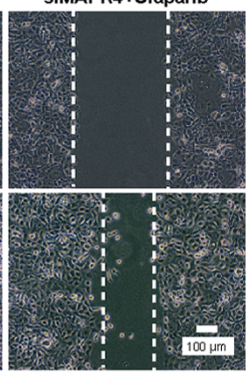

C

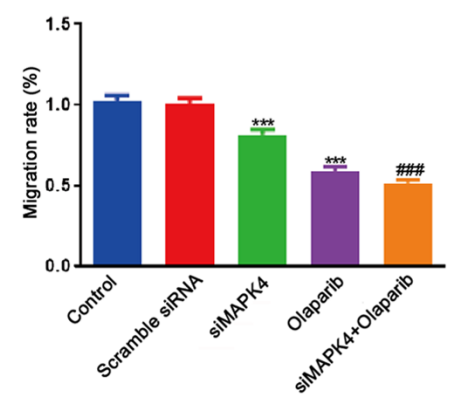

D

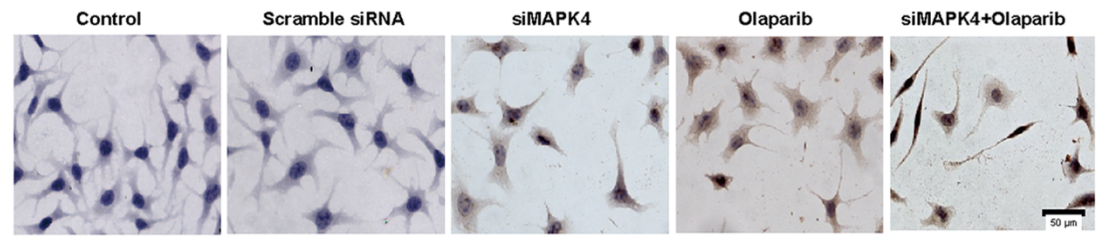

E

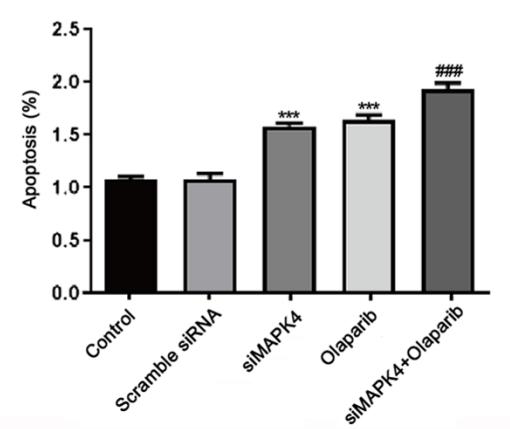

Figure 3. siMAPK4 enhances the sensitivity of TNBC cells to the poly ADP-ribose polymerase-1 inhibitor olaparib. Cells were seeded in 6-well plates overnight, and transfected with scramble siRNA and siMAPK4 plasmids for $48 \mathrm{~h}$. Subsequently, cells were treated with olaparib for $24 \mathrm{~h}$. (A) A Cell Counting Kit-8 assay was performed to test the cell viability. (B) The transfected cells were cultured, and the medium was changed with RPMI-1640 medium the following day. Next, the wound healing assay was performed. Scale bar, $100 \mu \mathrm{m}$. (C) The migration rate was calculated from the wound healing assay. (D) The apoptotic rate of cells treated with control, siRNA-scramble, siMAPK4, olaparib and a combination of siMAPK4 + olaparib was tested using the TUNEL assay. Scale bar, $50 \mu \mathrm{m}$. (E) Statistical analysis of the results of the TUNEL assay. ${ }^{* * * *} \mathrm{P}<0.001$ vs. control; ${ }^{\# \# /} \mathrm{P}<0.001$ vs. olaparib. siMAPK4, siRNA-MAPK4; siRNA, small interfering RNA.

siMAPK4 and control groups. The results demonstrated that MAPK4 could only phosphorylate $\mathrm{AKT}^{\mathrm{T} 308}$ (Fig. 4A). Thus, it was concluded that MAPK4 could exert its DNA damage and repair function by phosphorylating $\mathrm{AKT}^{\mathrm{T} 308}$.

Subsequently, the phosphorylation of $\mathrm{AKT}^{\mathrm{T} 308}$ was measured in MAPK4-overexpressing HCC1937 cells. The results indicated that MAPK4 overexpression increased the expression level of p-AKT ${ }^{\mathrm{T} 308}$ (Fig. 4B). HCC1937 cells were also transfected with the AKT-CA plasmid at the AKT ${ }^{\mathrm{T} 308}$ site to upregulate AKT phosphorylation (Fig. 4C and D). It was also identified that siMAPK4 increased the expression level of $\gamma \mathrm{H} 2 \mathrm{AX}$ (marker of a double-stranded break) (Fig. 4E). Moreover, siMAPK4 decreased the expression level of RAD51 and the phosphorylation of DNA-PK (Fig. 4F), which reflected DNA damage repair. These effects were blocked by AKT-CA. Collectively, these results indicated that MAPK4 exerted its DNA damage repair function by regulating the AKT signaling pathway. Moreover, olaparib aggravated the function of
siMAPK4, but weakened the function of AKT by lowering RAD51 and p-DNA-PK expression in the siMAPK4 group and the combination of siMAPK4 + AKT-CA group (Fig. 4F). These effects resulted in failure of DNA damage repair. Furthermore, the cells underwent apoptosis, as evident by the decrease in the expression level of Bcl-2 and the increase in that of Bax and cleaved-casp-3 when treated with olaparib and siMAPK4 (Fig. 4G).

\section{Discussion}

TNBC is intractable due to the absence of HER-2, estrogen receptor and progesterone receptor expression, which renders the currently available drugs ineffective (24). Previous studies have reported that PARP1 inhibitors are highly effective against breast cancer type 1 and 2 susceptibility proteins (BRCA1/2) and MCF-7 and MDA-MB-231 cells (25-27). It has also been revealed that TNBC has a lower expression or mutation rate 
A

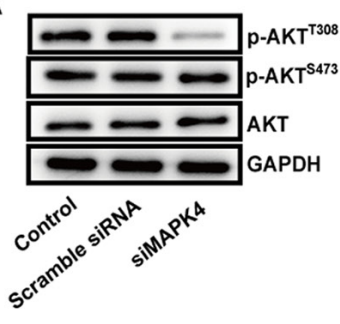

B

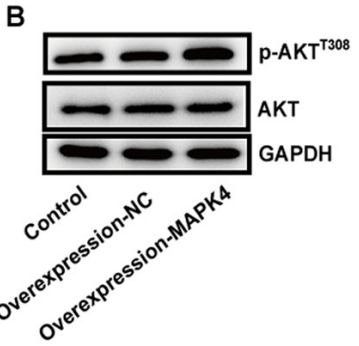

C

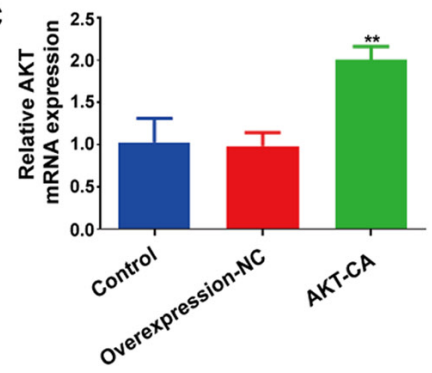

D

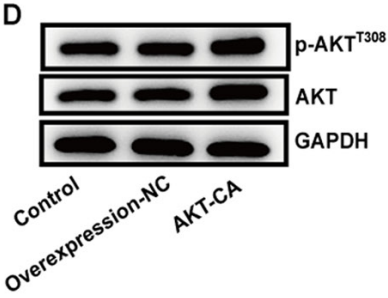

E
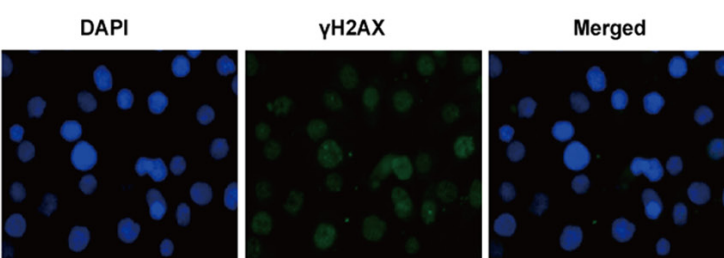

Control
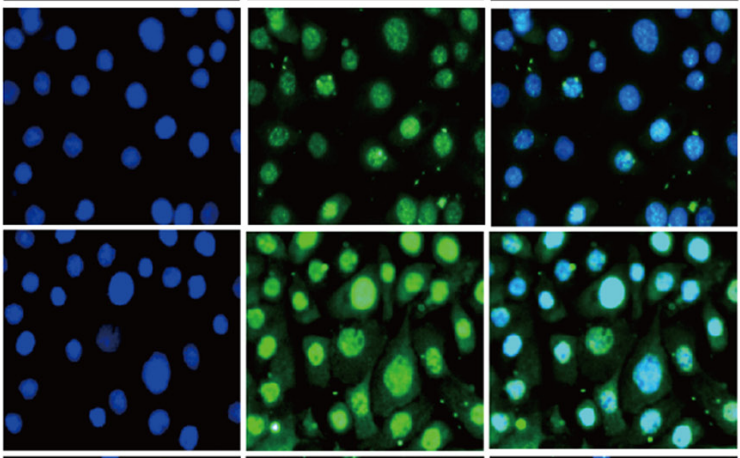

SiMAPK

$\mathbf{F}$
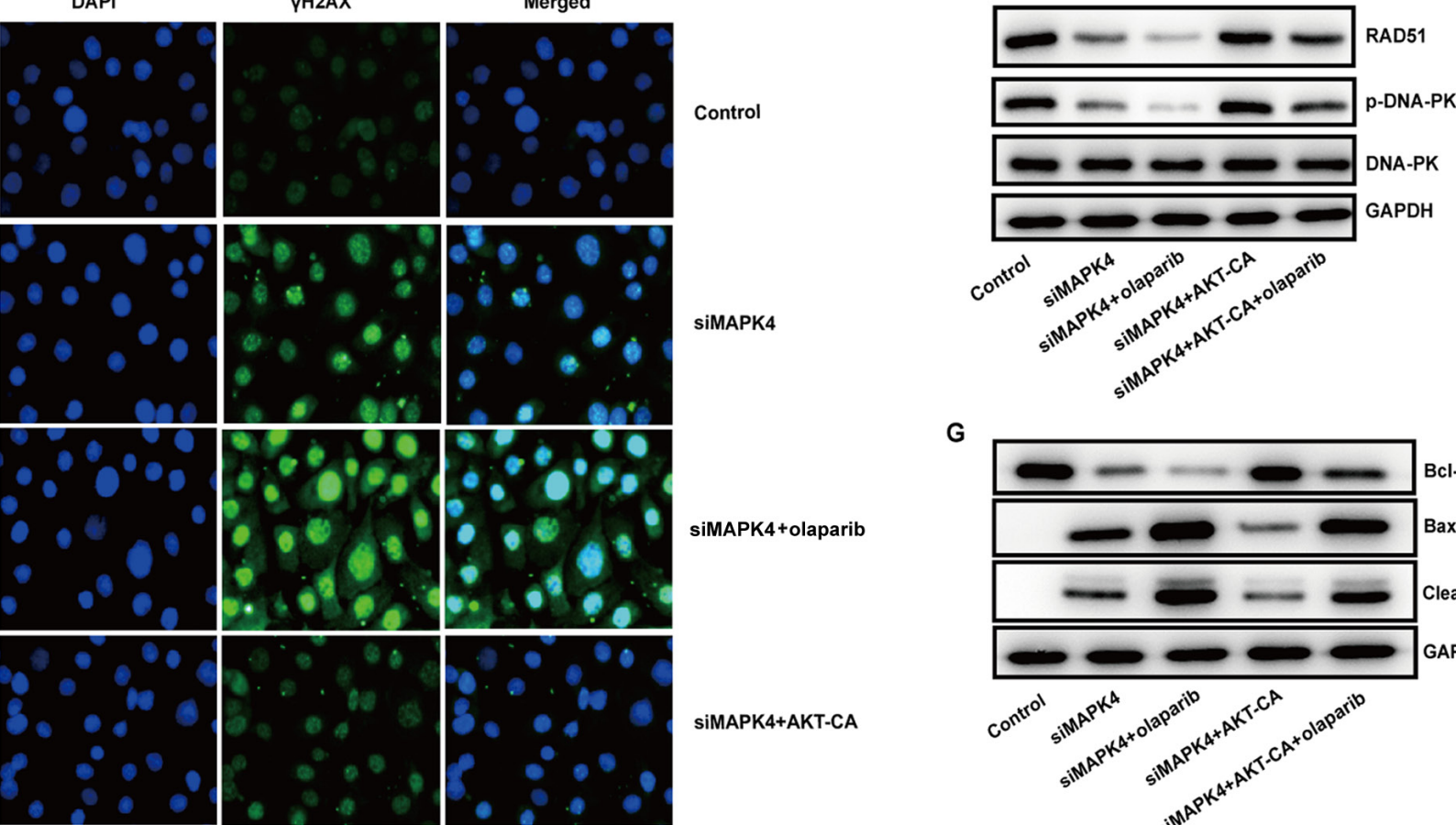

siMAPK4+olaparib

G
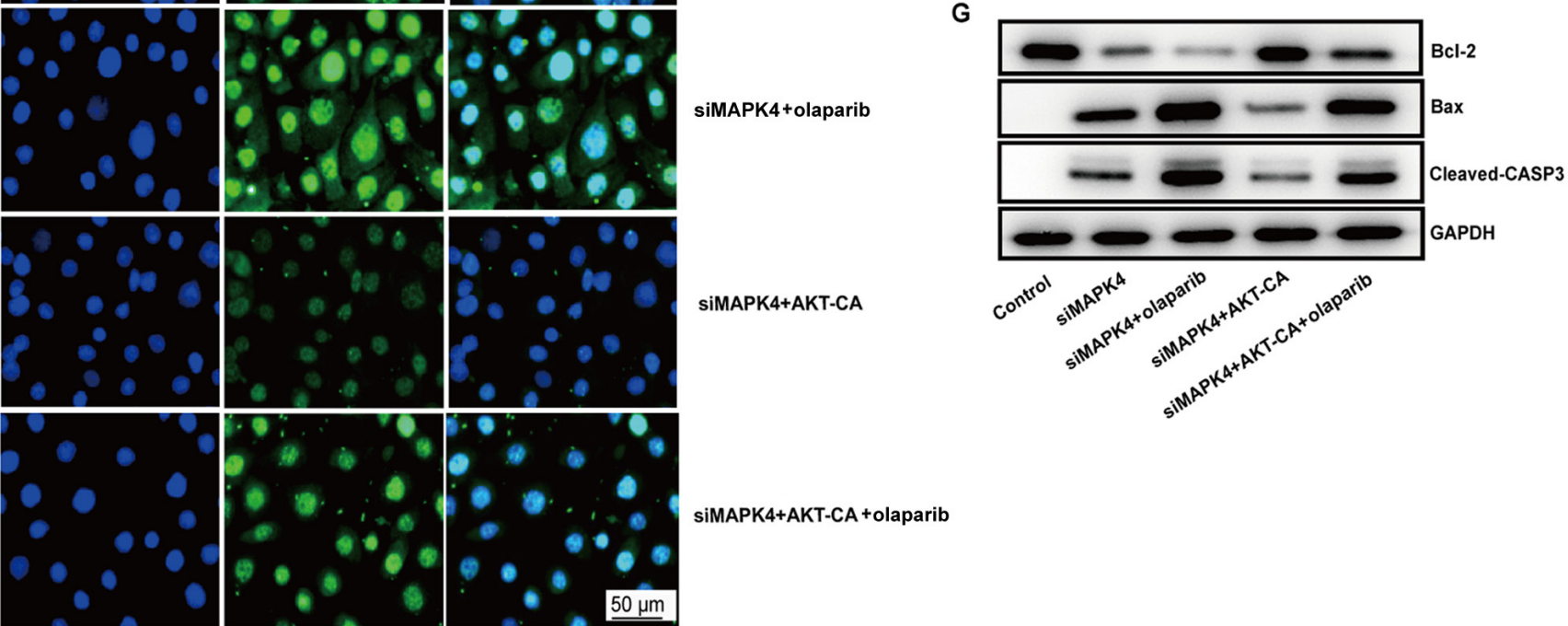

SIMAPK4+AKT-CA

siMAPK4+AKT-CA +olaparib

Figure 4. MAPK4 exerts its DNA damage repair function by regulating the AKT signaling pathway. (A) Western blotting was performed to test the protein expression levels of $\mathrm{p}-\mathrm{AKT}^{\mathrm{T} 308}$ and $\mathrm{p}-\mathrm{AKT}^{\mathrm{S} 473}$ in control cells, cells transfected with scramble siRNA and cells transfected with siMAPK4. (B) Protein expression level of p-AKT ${ }^{\mathrm{T} 308}$ in MAPK4-overexpressing cells vs. control cells was measured via western blotting. (C) mRNA expression level of AKT in AKT-CA cells was tested via reverse transcription-quantitative PCR. ${ }^{* *} \mathrm{P}<0.01$. (D) Western blotting was performed to examine the protein expression levels of p-AKT ${ }^{\mathrm{T} 308}$ and total AKT. (E) Immunofluorescence was used to test the protein expression level and location of $\gamma \mathrm{H} 2 \mathrm{AX}$ in the control, siMAPK, siMAPK with olaparib siMAPK, siMAPK + olaparib, siMAPK + AKT-CA and siMAPK + olaparib + AKT-CA groups. (F) Protein expression levels of RAD51 and p-DNA-PK were tested using western blotting in the control, siMAPK, siMAPK + olaparib, siMAPK + AKT-CA and siMAPK + olaparib + AKT-CA groups. (G) Protein expression levels of Bcl2 and Bax were tested via western blotting. AKT-CA, constitutively active AKT; NC, negative control; siMAPK4, siRNA-MAPK4; siRNA, small interfering RNA; $\gamma \mathrm{H} 2 \mathrm{AX}$, phosphorylated histone H2AX; casp, caspase; p-, phosphorylated; DNA-PK, DNA-dependent protein kinase catalytic subunit.

of BRCA1/2 (28). Hence, the use of PARP1 inhibitors for the treatment of TNBC is reasonable. In fact, PARP1 inhibitors, such as olaparib, have demonstrated high effectiveness against TNBC. However, the extensive use of PARP1 inhibitors can lead to the occurrence of resistance (29), partly due to the restoration of homologous recombination and upregulation of the ataxia-telangiectasia-mutated-and-Rad3-related kinase/checkpoint kinase 1 signaling pathway (30). Thus, it is important to identify other drugs or genes that can overcome this resistance.
MAPK4 is an oncogene that is upregulated in numerous types of cancer and contributes to poor prognosis. For instance, in cervical cancer, high MAPK4 mRNA expression is associated with a lower survival rate (31). The present data indicated that MAPK4 was highly expressed in patients with TNBC, suggesting that downregulating MAPK4 expression may enhance the effect of PARP1 inhibitors on TNBC. To verify this, the current study first determined the expression level of MAPK4 in TNBC tissues and cell lines. The results identified a higher MAPK4 expression in TNBC tissues and 
cell lines (MDA-MB-468, HCC1937 and MDA-MB-231) compared with that in normal tissues and cells (MCF-10A). Furthermore, MAPK4 overexpression promoted the proliferation of HCC1937 cells, while knockdown of MAPK4 inhibited cell viability. Next, the effect of combination of siMAPK4 and olaparib was determined using cell viability, wound healing and TUNEL assays. It was observed that MAPK4 knockdown in combination with olaparib treatment elicited a markedly greater inhibitory effect on cell proliferation, migration and apoptosis compared with either treatment alone.

Subsequently, the present study investigated the mechanism underlying how siMAPK4 enhances the sensitivity of TNBC cells to olaparib. Previous studies have reported that MAPK4 can phosphorylate AKT at T308 and S473 without influencing total AKT levels, thereby enhancing the efficacy of PARP1 inhibitors by suppressing the repair of radiation-induced DNA damage in cervical cancer cell lines $(9,31)$. Nevertheless, the current data indicated that MAPK4 could only activate $\mathrm{AKT}^{\mathrm{T} 308}$ in HCC1937 cells. It was also found that the expression levels of RAD51 and p-DNA-PK, which reflected the DNA damage repair, were inhibited by the PARP1 inhibitor and siMAPK. Moreover, the high $\gamma \mathrm{H} 2 \mathrm{AX}$ expression indicated that the PARP1 inhibitor and siMAPK could increase the double-stranded breaks. The present results also indicated that cells became apoptotic, due to the decreased expression level of Bcl-2 and increased Bax and cleaved-casp-3 expression when cells were treated with olaparib and siMAPK in the siMAPK4 + olaparib group.

Currently, there are several clinical trials investigating the role of olaparib and BRCA1/2 mutations in breast and ovarian cancer $(32,33)$. The present study demonstrated that siMAPK4 can enhance the sensitivity of TNBC cells to the PARP1 inhibitor by inhibiting $\mathrm{AKT}^{\mathrm{T} 308}$ phosphorylation and DNA repair, thereby promoting cell apoptosis in TNBC with BRCA1/2 mutations.

While the present study revealed that siMAPK4 can enhance the sensitivity of HCC1937 cells to a PARP1 inhibitor, olaparib, and identified the underlying mechanism in vitro, these results require further verification in animal models and human trials. Additionally, although it is known that MAPK4 can phosphorylate AKT at both T308 and S473 in bladder urothelial carcinoma, low-grade glioma, lung adenocarcinoma, cervical cancer, thyroid carcinoma and prostate cancer $(9,30,34)$, the present study found that only T308 was phosphorylated in TNBC cells and this was not further investigated. siRNAs can have poor stability and specificity, which may limit their usage in vivo (35). To solve these problems, in vivo experiments should be performed, and the use of nanoparticles should be considered to increase siRNA stability and ensure the effective delivery of these molecules to the appropriate target cells.

In conclusion, the present study demonstrated that MAPK4 was highly expressed in TNBC tissues and cell lines. Furthermore, knockdown of MAPK4 inhibited the proliferation of HCC1937 cells. By contrast, overexpression of MAPK4 increased the viability of HCC1937 cells. It was identified that knockdown of MAPK4 and treatment with the PARP1 inhibitor inhibited the proliferation and migration of HCC1937 cells by inhibiting DNA damage repair, increasing double-stranded breaks and promoting cell apoptosis. Therefore, MAPK4 may be another target for the treatment of TNBC in combination with PARP1 inhibitors.

\section{Acknowledgements}

Not applicable.

\section{Funding}

This work was funded by the President Foundation of Nanfang Hospital, Southern Medical University, Guangzhou, China (grant nos. 2016L007 and 2020C032).

\section{Availability of data and materials}

The datasets used and/or analyzed during the current study are available from the corresponding author on reasonable request.

\section{Authors' contributions}

$\mathrm{XZ}, \mathrm{SJ}, \mathrm{CY}$ and JD contributed to the study conception and design. XZ, SR and ZG performed the experiments. JG and ML contributed to the analysis and interpretation of data. XZ, SR and SJ contributed to the drafting of the manuscript. JD and $\mathrm{XZ}$ contributed to the project administration and writing, reviewing and editing the manuscript. JD and XZ confirm the authenticity of all the raw data. All authors contributed to the critical revision of the manuscript. All authors read and approved the final manuscript.

\section{Ethics approval and consent to participate}

Not applicable.

\section{Patient consent for publication}

Not applicable.

\section{Competing interest}

The authors declare that they have no competing interests.

\section{References}

1. Bray F, Ferlay J, Soerjomataram I, Siegel RL, Torre LA and Jemal A: Global cancer statistics 2018: GLOBOCAN estimates of incidence and mortality worldwide for 36 cancers in 185 countries. CA Cancer J Clin 68: 394-424, 2018.

2. World Health Organization (WHO): Cancer. https://www.who. int/news-room/fact-sheets/detail/cancer. Accessed March 3, 2021.

3. Gluz O, Liedtke C, Gottschalk N, Pusztai L, Nitz U and Harbeck N: Triple-negative breast cancer-current status and future directions. Ann Oncol 20: 1913-1927, 2009.

4. Foulkes WD, Smith IE and Reis-Filho JS: Triple-negative breast cancer. N Engl J Med 363: 1938-1948, 2010.

5. Vagia E, Mahalingam D and Cristofanilli M: The landscape of targeted therapies in TNBC. Cancers (Basel) 12: 916, 2020.

6. Coulombe P and Meloche S: Atypical mitogen-activated protein kinases: Structure, regulation and functions. Biochim Biophys Acta 1773: 1376-1387, 2007.

7. Lee BD, Park JM, Lee YM, Moon ES, Jeong HJ, Chung YI and Rim HD: A pilot study for discovering candidate genes of chromosome 18q21 in methamphetamine abusers: Case-control association study. Clin Psychopharmacol Neurosci 12: 54-64, 2014. 
8. Cancer Genome Atlas Research Network; Weinstein JN, Collisson EA, Mills GB, Shaw KR, Ozenberger BA, Ellrott K, Shmulevich I, Sander C and Stuart JM: The cancer genome atlas pan-cancer analysis project. Nat Genet 45: 1113-1120, 2013

9. Wang W, Shen T, Dong B, Creighton CJ, Meng Y, Zhou W, Shi Q, Zhou H, Zhang Y, Moore DD and Yang F: MAPK4 overexpression promotes tumor progression via noncanonical activation of AKT/mTOR signaling. J Clin Invest 129: 1015-1029, 2019.

10. Feng Y, Zhang L, Wu J, Khadka B, Fang Z, Gu J, Tang B, Xiao R, Pan G and Liu J: CircRNA circ_0000190 inhibits the progression of multiple myeloma through modulating miR-767-5p/MAPK4 pathway. J Exp Clin Cancer Res 38: 54, 2019.

11. He J, Huang Z, He M, Liao J, Zhang Q, Wang S, Xie L, Ouyang L, Koeffler HP, Yin D and Liu A: Circular RNA MAPK4 (circ-MAPK4) inhibits cell apoptosis via MAPK signaling pathway by sponging miR-125a-3p in gliomas. Mol Cancer 19: $17,2020$.

12. Piscitello D, Varshney D, Lilla S, Vizioli MG, Reid C, Gorbunova V, Seluanov A, Gillespie DA and Adams PD: AKT overactivation can suppress DNA repair via p70S6 kinase-dependent downregulation of MRE11. Oncogene 37: 427-438, 2018

13. Zhang Y, Xie C, Li A, Vizioli MG, Reid C, Gorbunova V, Seluanov A, Gillespie DA and Adams PD: PKI-587 enhances chemosensitivity of oxaliplatin in hepatocellular carcinoma through suppressing DNA damage repair pathway (NHEJ and HR) and PI3K/AKT/mTOR pathway. Am J Transl Res 11: 5134-5149, 2019.

14. Ali R, Rakha EA, Madhusudan S and Bryant HE: DNA damage repair in breast cancer and its therapeutic implications. Pathology 49: 156-165, 2017.

15. Gallmeier E and Kern SE: Absence of specific cell killing of the BRCA2-deficient human cancer cell line CAPAN1 by poly(ADP-ribose) polymerase inhibition. Cancer Biol Ther 4 703-706, 2005

16. Kim G, Ison G, McKe AE, Zhang H, Tang S, Gwise T, Sridhara R, Lee E, Tzou A, Philip R, et al: FDA approval summary: Olaparib monotherapy in patients with deleterious germline BRCA-mutated advanced ovarian cancer treated with three or more lines of chemotherapy. Clin Cancer Res 21: 4257-4261, 2015.

17. Balasubramaniam S, Beaver JA, Horton S, Fernandes LL, Tang S, Horne HN, Liu J, Liu C, Schrieber SJ, Yu J, et al: FDA approval summary: Rucaparib for the treatment of patients with deleterious BRCA mutation-associated advanced ovarian cancer. Clin Cancer Res 23: 7165-7170, 2017.

18. Noordermeer SM and van Attikum H: PARP inhibitor resistance: A tug-of-war in BRCA-mutated cells. Trends Cell Biol 29: 820-834, 2019.

19. Chandrashekar DS, Bashel B, Balasubramanya SAH, Creighton CJ, Ponce-Rodriguez I, Chakravarthi BVSK and Varambally S: UALCAN: A portal for facilitating tumor subgroup gene expression and survival analyses. Neoplasia 19 649-658, 2017.

20. Lin H, Li N, He H, Ying Y, Sunkara S, Luo L, Lv N, Huang D and Luo Z: AMPK inhibits the stimulatory effects of TGF- $\beta$ on Smad $2 / 3$ activity, cell migration, and epithelial-to-mesenchymal transition. Mol Pharmacol 88: 1062-1071, 2015.

21. Jiang S, Wang Y, Luo L, Shi F, Zou J, Lin H, Ying Y, Luo Y, Zhan Z, Liu P, et al: AMP-activated protein kinase regulates cancer cell growth and metabolism via nuclear and mitochondria events. J Cell Mol Med 23: 3951-3961, 2019.
22. Livak KJ and Schmittgen TD: Analysis of relative gene expression data using real-time quantitative PCR and the 2(-Delta Delta C(T)) method. Methods 25: 402-408, 2001.

23. Kwon KY, Jan JH, Kwon SY, Cho CH, Oh HK and Kim SP Cadmium induced acute lung injury and TUNEL expression of apoptosis in respiratory cells. J Korean Med Sci 18: 655-662, 2003.

24. Lyons TG: Targeted therapies for triple-negative breast cancer. Curr Treat Options Oncol 20: 82, 2019.

25. Bryant HE, Schultz N, Thomas HD, Parker KM, Flower D, Lopez E, Kyle S, Meuth M, Curtin NJ and Helleday T: Specific killing of BRCA2-deficient tumours with inhibitors of poly(ADP-ribose) polymerase. Nature 434: 913-917, 2005.

26. Dziadkowiec KN, Gąsiorowska E, Nowak-Markwitz E and Jankowska A: PARP inhibitors: Review of mechanisms of action and BRCA1/2 mutation targeting. Prz Menopauzalny 15: 215-219, 2016.

27. Lee JM, Ledermann JA and Kohn EC: PARP inhibitors for BRCA1/2 mutation-associated and BRCA-like malignancies. Ann Oncol 25: 32-40, 2014

28. Ryu JM, Choi HJ, Kim I, Nam SJ, Kim SW, Yu J, Lee SK, Choi DH, Park YH, Kim JW, et al: Prevalence and oncologic outcomes of BRCA 1/2 mutations in unselected triple-negative breast cancer patients in Korea. Breast Cancer Res Treat 173 385-395, 2019.

29. D'Andrea AD: Mechanisms of PARP inhibitor sensitivity and resistance. DNA Repair (Amst) 71: 172-176, 2018.

30. Mak JPY, Ma HT and Poon RYC: Synergism between ATM and PARP1 inhibition involves DNA damage and abrogating the $\mathrm{G}_{2}$ DNA damage checkpoint. Mol Cancer Ther 19: 123-134, 2020.

31. Tian S, Lou L, Tian M, Lu G, Tian J and Chen X: MAPK4 deletion enhances radiation effects and triggers synergistic lethality with simultaneous PARP1 inhibition in cervical cancer. J Exp Clin Cancer Res 39: 143, 2020.

32. Tutt A, Robson M, Garber JE, Domchek SM, Audeh MW, Weitzel JN, Friedlander M, Arun B, Loman N, Schmutzler RK, et al: Oral poly(ADP-ribose) polymerase inhibitor olaparib in patients with BRCA1 or BRCA2 mutations and advanced breast cancer: A proof-of-concept trial. Lancet 376: 235-244, 2010.

33. Audeh MW, Carmichael J, Penson RT, Friedlander M, Powell B, Bell-McGuinn KM, Scott C, Weitzel JN, Oaknin A, Loman N, et al: Oral poly(ADP-ribose) polymerase inhibitor olaparib in patients with BRCA1 or BRCA 2 mutations and recurrent ovarian cancer: A proof-of-concept trial. Lancet 376: 245-251, 2010.

34. Shen T, Wang W, Zhou W, Coleman I, Cai Q, Dong B, Ittmann MM, Creighton CJ, Bian Y, Meng Y, et al: MAPK4 promotes prostate cancer by concerted activation of androgen receptor and AKT. J Clin Invest 131: e135465, 2021.

35. Cho YS, Lee GY, Sajja HK, Qian W, Cao Z, He W, Karna P, Chen X, Mao H, Wang YA and Yang L: Targeted delivery of siRNA-generating DNA nanocassettes using multifunctional nanoparticles. Small 9: 1964-1973, 2013.

This work is licensed under a Creative Commons Attribution-NonCommercial-NoDerivatives 4.0 International (CC BY-NC-ND 4.0) License. 